Pecvnia, 7 (2008), pp. 227-256

\title{
El mercado de trabajo femenino en las empresas de economía social de Andalucía (España). Un análisis econométrico del perfil de la mujer trabajadora
}

\author{
$M^{a}$ Genoveva Millán Vázquez de la Torre \\ gmillan@etea.com \\ Universidad de Córdoba \\ Fac. de Ciencias Económicas y Empresariales \\ Escritor Castilla Aguayo, 4 \\ 14004 Córdoba (España) \\ Amparo Melián Navarro \\ amparo.melian@umh.es \\ Universidad Miguel Hernández \\ Esc. Politécnica Superior de Orihuela \\ Ctra. de Beniel km. 3,2 \\ 03312 Orihuela-Alicante (España)
}

Aceptado: Mayo 2009

En los últimos años se ha producido un incremento notable del número de mujeres que acceden al mercado de trabajo en España. Se observan, no obstante, diferencias significativas por comunidades autónomas, siendo Andalucía una de las menos favorecidas. En este estudio se analiza, el mercado de trabajo femenino en las empresas de Economía Social de la comunidad autónoma Andaluza, por ser ésta la que agrupa más del $25 \%$ de empresas de Economía Social a nivel nacional. Se trata con ello de conocer cómo está evolucionando
In recent years there has been a notable increase in the number of women entering the labour market in Spain. There are, however, significant differences in autonomous regions, Andalusia being one of the least favoured. This study examines the female labour market in companies of Social Economy of Andalusia, since this where over $25 \%$ of these Spanish companies are located. The job market for women is studied with the aim of identifying the most determinant factors influencing 
el mercado laboral femenino andaluz con la finalidad de determinar cuáles son los factores más determinantes que influyen en la demanda y en la oferta de empleo femenino de acuerdo con esta tipología de empresas (cooperativas y sociedades laborales) utilizando, para realizar dicha investigación, un modelo econométrico para definir el perfil de la mujer andaluza trabajadora.

Palabras clave: Andalucía, empleo femenino, empresas de economía social, mercado de trabajo. demand and supply of female employment in this type of business (industrial cooperatives and similar). Econometric models are employed to define the profile of working women in the South of Spain.

Key words: Andalusia, female employment, social economy enterprises, work-market.

\section{INTRODUCCIÓN}

La importancia de la Economía Social en Andalucía -como modelo empresarial integrado por empresas de carácter privado que actúan en el mercado produciendo bienes y servicios, que se caracterizan porque en la distribución del beneficio y el proceso de toma de decisiones interno predomina el factor humano frente al capital (Barea 1991; Monzón y Defourny 1992; Barea y Monzón 1996) - es una realidad contrastada durante la última década. Así se evidencia en las bases de datos de las series temporales de las principales magnitudes de la economía andaluza, en las cuales puede verse el peso que tienen las cooperativas ${ }^{1}$ y las sociedades laborales $^{2}$ (siendo estas dos formas jurídicas las principales componentes de la Economía Social), y donde estas empresas actúan como agentes capaces de aglutinar la función económica de crear riqueza con eficiencia y la función social de distribuirla con equidad.

La economía andaluza mantiene posiciones bastante discretas respecto a la media nacional en magnitudes como la productividad y el

1 Según la Ley $3 / 2002$, de 16 de diciembre, por la que se modifica la Ley $2 / 1999$, de 31 de marzo, de Sociedades Cooperativas Andaluzas, se define Cooperativa como una sociedad participativa que asocia a personas físicas o jurídicas que tienen intereses o necesidades socioeconómicas comunes, para cuya satisfacción y en interés de la comunidad realizan cualquier actividad empresarial, con arreglo a los principios o disposiciones de dicha Ley.

2 Se define Sociedad Laboral como una empresa en la que la mayoría del capital social es propiedad de los trabajadores que prestan personal y directamente en ella servicios retribuidos con una relación laboral por tiempo indefinido. Su calificación corresponde al MTAS (Ministerio de Trabajo y Asuntos Sociales). Se administran igual que una Sociedad Anónima o una Sociedad Limitada, según sean Sociedades Anónimas Laborales (SAL) o Sociedades Limitadas Laborales (SLL). En ellas ningún socio puede ostentar más de un tercio del capital social, $50 \%$ si es ente público. 
empleo -especialmente el femenino-, ocupando algunas provincias andaluzas los primeros puestos de la lista nacional mensual de desempleo. A pesar de ello se ha producido una evolución creciente de la Economía Social, a nivel nacional y andaluz, que proyecta unas perspectivas positivas, sobre todo en términos de generación de empleo femenino.

De la importancia de las empresas de Economía Social en España y en Andalucía dan muestra algunos datos que ofrecen instituciones como el Ministerio de Trabajo y Asuntos Sociales, la Confederación de Entidades para la Economía Social en Andalucía (CEPES Andalucía), o la Agencia Estatal de Administración Tributaria (AEAT) en las declaraciones del Impuesto de Sociedades.

Así, a finales de 2007 existían en España 43.655 empresas de Economía Social (ES), de las cuales 20.780 eran cooperativas y 22.885 sociedades laborales (47,6 y $52,4 \%$ respectivamente). La comunidad autónoma con mayor número de empresas de ES es Andalucía con 11.315 (el $25,9 \%$ del total nacional), seguida a notable distancia por Cataluña, con 4.969 empresas, y la Comunidad Valenciana, con 4.323 empresas. Entre las tres comunidades agrupan el 47,2\% de las empresas de Economía Social a nivel nacional (CEPES 2007a). El hecho de que un 25,9\% de las empresas de ES se hallen en Andalucía denota cierta relevancia de esta forma empresarial en la comunidad autónoma, pues Andalucía cuenta con el $18 \%$ de la población, es la sede social del $15 \%$ de las empresas y representa el $13,5 \%$ del total de la producción a nivel nacional.

En 2007 de las 11.315 empresas de ES andaluzas inscritas, 5.596 son cooperativas y 5.719 sociedades laborales, 49,5 y $50,5 \%$ respectivamente. En los últimos diez años el número de empresas ha crecido en un $92,9 \%$, siendo las sociedades laborales la forma jurídica que mayor incremento ha experimentado (en 1997 existían 5.114 cooperativas y 751 sociedades laborales). De hecho no es hasta 2005 cuando se igualan los porcentajes de ambas formas jurídicas, pues al inicio del periodo el $87,2 \%$ eran sociedades cooperativas y sólo el $12,8 \%$ sociedades laborales. Los cambios normativos en las sociedades laborales han propiciado el desarrollo de éstas en los últimos años.

En 2007 se dieron de alta 1.031 empresas de ES en Andalucía (358 cooperativas y 673 sociedades laborales), un 70\% más de las que se crearon hace una década (608 empresas, 516 cooperativas frente a sólo 92 sociedades laborales). Tras un incremento generalizado desde 1997 hasta 2002 con cifras que superaron las 2.200 empresas en 2002 y 2003, los años 
siguientes han sido de menor crecimiento hasta llegar a las cifras actuales (en torno a las 1.000 empresas) que fueron muy similares a las del año anterior 2006. En las provincias que más se han creado son Sevilla, Málaga y Córdoba, sumando entre ambas el $55,1 \%$, sólo las dos primeras ya suman el $42,3 \%$. De todas las empresas creadas el $65 \%$ lo hizo como sociedad laboral y el 35\% como cooperativa, lo que según la Consejería de Innovación, Ciencia y Empresa estima en una generación de empleo de 8.200 puestos directos.

En cuanto al crecimiento en el periodo 1997-2007 Almería ha triplicado la cifra de 1997, llegando a tener en la actualidad 1.282 empresas registradas, Málaga con 1.635 ha multiplicado por 2,5. Jaén es la que menor crecimiento ha tenido en los diez años, un 44\%, con 1.180 empresas censadas en 2007.

Para conocer el peso de la Economía Social sobre la Economía Global es importante conocer qué porcentaje de empresas pertenece a este sector respecto al total de empresas existentes. En 2007 el 13,4 por mil de las empresas españolas pertenecían al sector de la ES. Murcia es junto con Andalucía las comunidades autónomas donde mayor es el peso de las empresas de ES sobre el total, en Murcia es del 27,8\% y en Andalucía del 22,5\%. En 2007 en Andalucía las empresas de ES creadas representaron el 4,5\% sobre el total de todas las empresas creadas en dicho año. Por provincias destaca Jaén y Huelva donde la fórmula cooperativa o de sociedad laboral fue adoptada por una de cada diez nuevas empresas constituidas (Consejería de Innovación, Ciencia y Empresa 2007).

Las empresas de economía social en España dan empleo a 442.326 trabajadores (317.542 en cooperativas y 124.784 en sociedades laborales, 72 y $28 \%$ respectivamente). Esto supone un incremento del $56,1 \%$ respecto al empleo de hace una década, donde éste fue de 283.392 trabajadores (de los cuales 227.609 estaban empleados en cooperativas y 55.783 en sociedades laborales) (MTAS 2008). Por comunidades autónomas han crecido de forma importante en Murcia y Andalucía. Andalucía con 90.016 empleos (60.982 en cooperativas y 29.034 en sociedades laborales, 68 y $32 \%$ respectivamente) se coloca en primera posición en cuanto a número de empleos en empresas de economía social (20,4\% total nacional), duplicando la cifra de 1997 de 44.920 empleos (39.168 en cooperativas, 5.752 en sociedades laborales). Ello es muy significativo porque este crecimiento le ha permitido pasar de ser la tercera comunidad autónoma en empleo en empresas de ES en 1997 a ser la primera en la actualidad. Por el contrario Cataluña que en 1997 era la primera ha dejado de estar entre 
las posiciones relevantes del ranking debido al poco crecimiento $(13,7 \%$, el menor de todas las comunidades autónomas) experimentado. El segundo y tercer lugar lo ocupan respectivamente el País Vasco (15,7\%) y C. Valenciana $(14,6 \%)$, con crecimientos en ambos en torno al 50\% (MTAS 2007).

Cuadro 1: Empleo generado en las empresas de Economía Social según su forma jurídica
\begin{tabular}{|l|r|r|r|r|c|}
\hline \multicolumn{1}{|c|}{ Año $\mathbf{2 0 0 7}$} & Cooperativas & $\begin{array}{c}\text { Sociedades } \\
\text { Laborales }\end{array}$ & Total & $\begin{array}{c}\text { \% sobre } \\
\text { España }\end{array}$ & Ranking \\
\hline España & 317.542 & 124.784 & 442.326 & & \\
\hline Andalucía & 60.982 & 29.034 & 90.016 & 20,35 & $1^{\mathbf{o}}$ \\
\hline País Vasco & 56.039 & 13.462 & 69.501 & 15,71 & $2^{\mathbf{o}}$ \\
\hline Com. Valenciana & 54.082 & 10.482 & 64.564 & 14,60 & $3^{\mathbf{0}}$ \\
\hline Cataluña & 42.359 & 10.384 & 53.193 & 12,03 & $4^{\mathbf{0}}$ \\
\hline La Rioja & 1.800 & 431 & 2.231 & 0,50 & $17^{\mathbf{0}}$ \\
\hline
\end{tabular}

Fuente: Elaboración propia a partir de datos MTAS (2007).

Los datos de la AEAT indican que las empresas de Economía Social declararon en el año 2007 una producción superior a los 60.000 millones de euros, de los cuales el $82,3 \%$ lo generaron las cooperativas y el $17,7 \%$ restante las Sociedades Laborales. El $17,5 \%$ de la producción se obtuvo en Andalucía, el 15\% en el País Vasco, el 14\% en Cataluña 14\% y el $11 \%$ en la Comunidad de Madrid.

Por sectores de actividad, el $9 \%$ de las empresas de Economía Social pertenecen al primario, el $20 \%$ a la construcción, otro $21 \%$ a la industria y el $50 \%$ restante al sector servicios, dentro del cual comercio y hostelería supone el $26,2 \%$. Donde existen menos empresas es en agricultura y pesca, transporte, almacenamiento y comunicaciones. Las cifras anteriores distan de las presentadas en 1997, donde el $25 \%$ de las empresas se encuadraban en el sector primario (agricultura), el $9 \%$ en la construcción, el $27 \%$ en la industria y el $39 \%$ en servicios (CEPES 2007a). En cuanto a la distribución de empelo por sectores de actividad es hoy en día mayoritario en el sector servicios ${ }^{3}(50 \%)$, seguido por el industrial $(20 \%)$, la construcción (16\%) y la agricultura (14\%), frente a los valores de 1997 donde las cifras fueron respectivamente del 46, 29, 9 y $16 \%$. Observamos

3 Comercio y hostelería generan el $48 \%$ de los empleos del sector servicios mientras que la intermediación financiera y las actividades inmobiliarias generan el $24 \%$. 
que aunque la distribución de empresas sí que ha cambiado sensiblemente este cambio no se ha trasladado tan proporcionalmente al empleo. Destacable es también, a la vista de los datos anteriores, la ruptura con el tópico que adjudicaba a la ES una vinculación directa con el sector agrario, cuando aquí se pone de manifiesto su vinculación con otros sectores económicos.

La distribución de empleos en cooperativas y en sociedades laborales es similar en los servicios y la industria $(70$ y $30 \%$, respectivamente). En la construcción son mayores los empleos en las sociedades laborales, mientras que en el sector agrario son mayores en las cooperativas.

En el ámbito de Andalucía, las empresas de Economía Social se encuentran presentes en 4 de cada 5 municipios, lo que da idea del equilibrio en su distribución territorial. Sevilla la provincia con mayor concentración de empresas de este tipo, pues cuenta con el 22,1\% del total regional, le siguen Málaga, con el 14,5\%, Córdoba, con el $12 \%$. Almería 11,3\%, Cádiz 10,7\%, Jaén 10,4\%, Huelva 9,6\% y Granada 9,4\%. Sevilla es también la provincia que más empleo genera con 19.194 trabajadores, lo que supone el $21,3 \%$ del total andaluz, seguida de Almería con 14.193 $(15,8 \%)$ y Málaga con 10.194 (12,1\%) (CEPES 2007a).

De las características socioeconómicas de los trabajadores de empresas andaluzas de Economía Social, según informe del CEPES (2007b) destacamos como las más significativas:

- El 28,2\% tiene más de tres años de antigüedad en la empresa

- El 62,5\% (3 de cada 5) tiene una edad inferior a 40 años, porcentaje que supera en 8 puntos a la media nacional

- El 92,3\% tiene nacionalidad española. Las provincias con mayor presencia de trabajadores extranjeros son Almería $(20,5 \%)$ y Huelva $(17,8 \%)$

- El $38,5 \%$ de los trabajadores son mujeres. La única provincia andaluza con mayoría de trabajadoras es Almería (52,2\% mujeres)

- El $86,4 \%$ de los trabajadores trabajan a tiempo completo

- El $54 \%$ tienen contratos indefinidos

- El $28,5 \%$ de los empleados tienen su centro de trabajo en empresas con más de 100 trabajadores. En Almería el porcentaje sube al $57,3 \%$ 
- El 2,1\% de los autónomos andaluces (7.440 personas) son socios trabajadores de cooperativas y cotizan a la Seguridad Social bajo el RETA (Régimen Especial de Trabajadores Autónomos), frente al 3,9\% de los autónomos nacionales que son socios trabajadores de cooperativas

- El 70,3\% de los autónomos andaluces pertenecen al sector servicios

\section{EL EMPLEO FEMENINO EN LAS EMPRESAS DE ECONOMÍA}

SOCIAL

Al analizar el empleo en las empresas de Economía Social hay tener en cuenta el tamaño de las mismas pues esto es determinante a la hora de generar puestos de trabajo. Así en las cooperativas de España el número medio de trabajadores es de 12,3, siendo el País Vasco el que posee una media mayor de 37,6 trabajadores frente a Cataluña que es la menor con 7,5 trabajadores, mientras que Andalucía posee 11,1 empleados de media en sus empresas cooperativas (CEPES 2007b). Por el contrario las Sociedades Laborales suelen ser empresas con menor número de trabajadores que las cooperativas siendo el número medio de trabajadores de 6,3 casi la mitad que el de las cooperativas. El País Vasco es la comunidad donde la Sociedades Laborales tienen mayor número de trabajadores 13,2 de media, casi un tercio del de las cooperativas para la misma región, teniendo las Sociedades Laborales andaluzas 6,2 trabajadores de media, y por lo tanto en línea con la media nacional (CEPES 2007b).

Con respecto al porcentaje de empleados en Economía Social frente al total de ocupados, nos encontramos que a nivel nacional sólo el 2,2\% de los trabajadores están empleados en empresas de Economía Social, lo que significa que 1 de cada 50 ocupados pertenecen a empresas de ES. Por comunidades destaca el País Vasco donde el 6,9\% de los empleados trabajan en empresas de Economía Social frente al 0,8\% de Baleares. En Andalucía que es la comunidad objeto de estudio, el 2,8\% de los empleados trabajan en cooperativas y sociedades laborales, empresas de ES. Otras comunidades que superan la media nacional son Murcia, Navarra, C. Valenciana, y Castilla-La Mancha.

Analizando el trabajo por sexos en las empresas de Economía Social (Gráfico 1), y según CEPES (2007b) nos encontramos que el 38,5\% 
de los trabajadores de dichas empresas en Andalucía son mujeres, cifra dos puntos porcentuales inferior a la media nacional (40,8 \%). En ninguna comunidad autónoma española el porcentaje del colectivo de trabajadoras femenino es superior al masculino, la comunidad que está más cerca de lograr la paridad en el empleo dentro de la Economía Social es Baleares donde el $49,5 \%$ de los trabajadores son mujeres frente al $50,5 \%$ de los varones, seguida del País Vasco el $44,2 \%$ son mujeres frente al $55,8 \%$, por el contrario y a la cola se encuentra Extremadura donde la disparidad entre el porcentaje de empleo masculino es de 70 frente al $30 \%$ del femenino (más del doble).

Gráfico 1: Trabajo por sexos en las Empresas de Economía Social (comunidades con mayor y menor disparidad entre ambos)

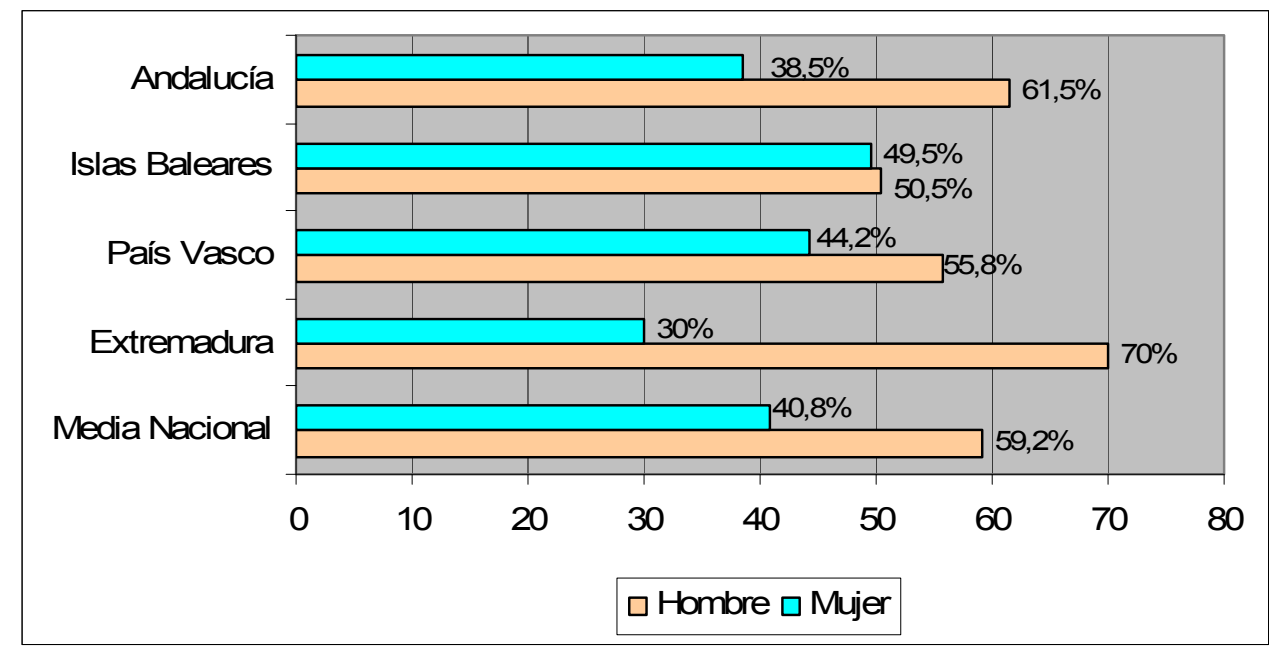

Fuente: Elaboración propia a partir de CEPES (2007b).

La evidencia empírica tras diversos estudios realizados en el sector de la Economía Social indica que la participación laboral de hombres y mujeres varía según el tipo de entidad de que se trate, cooperativa o sociedad laboral (Ribas 2004). El estudio de Ribas y Sajardo (2005) muestra que cuando se toman los datos de participación de mujeres en cooperativas y sociedades laborales de forma conjunta, los resultados son similares a las tasas de participación en el conjunto de la economía (39\% en economía social frente al 39,6\% del total de la población ocupada en España en el primer trimestre de 2005 según datos del Ministerio de Trabajo y Asuntos Sociales). Sin embargo, si se observan por separado los datos del trabajo de mujeres en cooperativas y sociedades laborales se aprecia 
una diferencia de 20 puntos porcentuales si se compara la participación de las mujeres en cooperativas y en sociedades laborales anónimas $(43,7 \%$ frente $24,0 \%$ ), y una diferencia de 15 puntos porcentuales si se compara cooperativas con sociedades laborales limitadas (43,7\% frente $29,0 \%$ ). Estas cifras revelan por tanto una participación mayor de mujeres en las cooperativas (4,1 punto superior) que en el total de la población ocupada en la economía española. Por otra parte también existen ciertas diferencias en cuanto a la tasa de empleo si se atiende a la clase de cooperativas. Así, las mujeres están sobrerepresentadas en las cooperativas sanitarias (78\% trabajadores femeninos), sanitarias (78\%), enseñanza (74\%) y consumidores y usuarios (73\%), y están subrepresentadas en las cooperativas del mar $(18 \%)$, de vivienda (26\%), de crédito (34\%), de explotación comunitaria de la tierra (34\%) y de transportistas (35\%) (MTAS 2007).

La diferencia de valores en el empleo femenino entre cooperativas y sociedades laborales se debe al diverso origen de ambos tipos de empresas. En las cooperativas el nexo de unión entre los socios y la motivación que les conduce a asociarse creando la empresa, es la ayuda mutua a diferentes niveles, social y/o económico, mientras que las sociedades laborales son sociedades mercantiles en las que la mayor parte del capital está en manos de los trabajadores. Las cooperativas trabajan en un plano mayor de igualdad y la forma de participación en la gestión de la sociedad es también mucho mayor.

Apelqvist (1996) apunta las semejanzas que existen entre los valores cooperativos y la forma en que las mujeres conceptualizan el trabajo. De hecho afirma que las culturas femeninas se caracterizan por la autoayuda, la confianza en sí mismo, la democracia, la igualdad, la equidad y la solidaridad. Las mujeres poseen un enfoque no jerárquico en relación a la organización del trabajo. Para ellas es importante tomar las decisiones de forma consensuada, pues se genera una mayor motivación y responsabilidad, al tiempo que se facilita su implementación de forma más rápida y fiable. Chávez (1996) afirma que las cooperativas, gracias a su estructura democrática, ofrecen a las mujeres los medios para adquirir experiencia y movilidad ascendente en la estructura de la empresa.

Un estudio realizado por Costa-Pau (2002) para la ciudad de Barcelona muestra la percepción de las mujeres en relación a su trabajo en una cooperativa. Así según los resultados de éste el $57 \%$ de las mujeres encuestadas admiten que el hecho de trabajar en una cooperativa tiene un interés especial frente a un 33\% que opinan lo contrario. Dicho interés radica en primer lugar en las relaciones laborales (un $77 \%$ ) seguido del 
interés por las relaciones personales (un 22\%) (sólo el $1 \%$ restante se refirió a motivaciones de tipo económico).

Si analizamos los datos de empleo en Economía Social comparándolos con el mercado de trabajo nacional nos encontramos que desde la promulgación de la Constitución Española de 1978, que supuso el punto de partida para superar la desigualdad que venía existiendo entre hombre y mujer hasta nuestros días, muchos han sido los avances efectuados por la mujer española a nivel social y laboral, alcanzando en la actualidad un $47,9 \%$ de tasa de actividad y un $11,6 \%$ de tasa de paro ${ }^{4}$, cifras aún lejanas de las cotas alcanzadas por las mujeres en otros países industrializados como, por ejemplo, Dinamarca (con una tasa de actividad del $60 \%$ ) o Suecia (con un $8 \%$ de tasa de paro).

Los cambios más importantes acaecidos en la sociedad se pueden resumir en tres: primero, en el mercado laboral se ha incrementado la tasa de actividad en 17 puntos en algo más de dos décadas (del 32,4\% del primer trimestre de 1987 al 49,7\% del mismo trimestre de 2008; segundo, en el sistema educativo la tasa de escolaridad de la mujer es superior en casi todas las edades a la de los varones, incluida la educación universitaria, excepto en las carreras técnicas; tercero, la disminución de la tasa de natalidad (de 2,2 hijos en los años 80 a 1,1 hijos en 2007 (INE 2008).

A pesar de la importancia de estos tres fenómenos, existe todavía en España, y con más agudeza en Andalucía, tanto en los sectores de actividad como en las profesiones, una fuerte segregación por sexos, y las mujeres siguen ocupando las categorías profesionales definidas con escasa cualificación, baja remuneración y menor prestigio social, siendo también el trabajo temporal el que más incidencia tiene en este colectivo.

Partiendo de estas premisas se ha realizado el presente estudio: Andalucía es la comunidad autónoma con mayor número de personas potenciales en edad de trabajar, es decir, entre 16 y 65 años (19.372.389 de personas ${ }^{5}$ ) (Cuadro 2), de las cuales algo menos de diez millones corresponde a población de mujeres activas, frente a 12.942.538 de varones activos. Es también la primera comunidad autónoma con mayor número de trabajadores empleados en empresas de Economía Social, siendo

4 Encuesta de Población Activa (EPA), primer trimestre 2008.

5 Datos del INE de marzo de 2008. 
también la segunda comunidad autónoma con mayor tasa de paro masculina y femenina (Cuadro 3) y la penúltima comunidad en tasa de actividad (Cuadro 4).

Cuadro 2: Población femenina según edad y situación laboral (miles de personas, en cursiva y paréntesis las cifras de hombres)

\begin{tabular}{|l|c|c|c|}
\hline \multicolumn{1}{|c|}{ Edad } & Nacional & Andalucía & Cataluña \\
\hline \multirow{2}{*}{ 16 ó mas años } & $19.372,4$ & $3.365,8$ & $3.081,4$ \\
& $(18.670,3)$ & $(3.259,5)$ & $(2.986,0)$ \\
\hline \multirow{2}{*}{ Activas/os } & $9.633,9$ & $1.555,7$ & $1.658,6$ \\
& $(12.942,5)$ & $(2.229,9)$ & $(2.166,8)$ \\
\hline \multirow{2}{*}{ Ocupadas/os } & $8.478,4$ & $1.257,9$ & $1.514,1$ \\
& $(11.923,9)$ & $(1.966,1)$ & $(2.020,4)$ \\
\hline \multirow{2}{*}{ Ocupadas/os en Economía Social } & 180,7 & 34,6 & 22,2 \\
& $(262,2)$ & $(55,4)$ & $(31,0)$ \\
\hline \multirow{2}{*}{ Paradas/os } & $1.156,6$ & 297,8 & 144,4 \\
& $(1.018,6)$ & $(263,7)$ & $(146,4)$ \\
\hline
\end{tabular}

* Se ha escogido la Comunidad de Cataluña para hacer la comparación, por ser la segunda comunidad con mayor porcentaje de empresas en Economía Social $(11,38 \%)$ y la cuarta en creación de empleo en Economía Social (12,03\%).

Fuente: Elaboración propia en base a datos del INE y MTAS primer trimestre de 2008.

Analizando el entorno económico nacional, según los datos del Instituto Nacional de Estadística (INE), se observan grandes diferencias entre comunidades autónomas. Así, se obtiene que la renta per cápita media de los españoles fue, en 2006 de 22.260 euros, un 10,2\% inferior a la renta de la Unión Europea. Sólo seis comunidades autónomas -Aragón, Baleares, Cataluña, Madrid, Murcia y País Vasco- superan la renta per cápita de la Unión Europea (que asciende a 23.700 euros). Además de estas cuatro regiones, otras tres -Aragón, Baleares y La Rioja- tienen una renta superior a la media española.

Tras Madrid (28.747 euros por habitante), se sitúan en la clasificación de las regiones más ricas el País Vasco (28.731 euros), y la Comunidad de Murcia (27.856 euros). A continuación se situó Cataluña (26.279 euros), Baleares (24.265 euros), Aragón (23.707 euros) y La Rioja (23.571 euros). En el otro extremo de la balanza se encuentra Extremadura (15.031 euros), Andalucía (17.401 euros) y Castilla-La Mancha (17.672 euros). Es decir, existen diez regiones más dos ciudades autónomas (Ceuta y Melilla) con un nivel de riqueza inferior a la media nacional. 
El indicador de riqueza (renta), la tasa de actividad y la tasa de paro están altamente correlacionadas ${ }^{6}$. Dada la disparidad existente entre las diversas comunidades autónomas, en el Cuadro 3 se muestra una síntesis de las comunidades con menor tasa de paro femenino (Madrid y Cataluña) y las dos con mayor tasa (Extremadura y Andalucía). Estas últimas, tal y como hemos señalado, tienen un nivel de renta per cápita muy inferior a la media nacional, estando en el vagón de cola del desarrollo económico de España, ya que uno de los principales indicadores de prosperidad de una zona es que tenga una tasa de desempleo inferior al $5 \%$ ( $\mathrm{y}$, en consecuencia, pleno empleo), objetivo alejado en la actualidad a nivel nacional, y aún más en estas dos comunidades.

La importante tasa de paro femenina en Andalucía y Extremadura y su diferencia superior en 7 puntos respecto del paro masculino justifica la necesidad y oportunidad de estudiar el mercado femenino en cada una de estas dos comunidades. En el presente trabajo se ha elegido Andalucía por tres razones:

- Porque tienen un mayor número de población femenina, potencialmente afectada por este problema.

- Porque está constituida por provincias bastantes dispares entre sí. Así, algunas capitales, como Málaga o Sevilla, se encuentran entre las ciudades españolas con mayor renta, y otras, como Jaén, en los últimos puestos de la clasificación nacional.

- Por ser la comunidad con mayor número de empresas de Economía Social.

Analizando el Cuadro 3 se puede observar que en todas las comunidades autónomas la tasa de paro femenina es superior a la masculina, siendo las diferencias más significativas en Extremadura con más de 12 puntos porcentuales, seguida de Andalucía, con más de 7 puntos. Sin embargo, en la Comunidad Catalana la diferencia es de tan sólo un 1,95 puntos, siendo la tasa de paro femenina $(8,71 \%)$ en esta comunidad similar a la media europea, que se situó entorno al $8 \%$. En España existen comunidades con bajas tasas de paro femenino (Madrid, Cataluña o Baleares), coincidentes con el mayor desarrollo económico, 
con un PIB más elevado; frente a otras, como Extremadura o Andalucía, en las que el sector primario sigue teniendo todavía un peso importante en la economía, y donde las mujeres de zonas rurales son los principales perceptores del subsidio agrario, con una elevada tasa de paro por la temporalidad del sector.

Fuente: Elaboración propia en base a datos del INE. Primer trimestre de 2008.

En el Cuadro 4 se presenta la tasa de actividad femenina en cuatro comunidades autónomas, las dos mejores y las dos peores del país en relación a dicha tasa de actividad femenina, pertenecientes a dos grupos donde las diferencias entre sí son más acusadas.

\begin{tabular}{|c|c|c|c|c|}
\hline \multicolumn{5}{|c|}{ Cuadro 4: Tasa de Actividad } \\
\hline & Ambos sexos & Varones & Mujeres & $\begin{array}{l}\text { Diferencia } \\
=|V-M|\end{array}$ \\
\hline Nacional & 59,35 & 69,32 & 49,73 & 19,59 \\
\hline Extremadura & 52,84 & 64,34 & 41,63 & 22,71 \\
\hline Andalucía & 57,14 & 68,41 & 46,22 & 22,19 \\
\hline Madrid & 63,83 & 72,61 & 55,69 & 16,92 \\
\hline Cataluña & 63,05 & 72,56 & 53,83 & 18,73 \\
\hline
\end{tabular}

Fuente: Elaboración propia a partir de los datos del INE. Primer trimestre de 2008.

En el Cuadro 4 se puede observar también que la tasa de actividad masculina es bastante superior a la femenina en todas las comunidades, siendo la diferencia superior a 18 puntos porcentuales en casi todas ellas, destacando, negativamente, Andalucía y Extremadura con un 22,19 y $22,71 \%$ respectivamente.

Si analizamos por tipo de contratos, en Andalucía, por cada 100 contratos a tiempo parcial que realizan los hombres hay 140 mujeres (MTAS 2007) que tienen la misma modalidad siendo su índice de 
feminización ${ }^{7}$ más elevado, ya que la mujer ha de compatibilizar el trabajo del hogar con el trabajo fuera de casa y la "mejor opción", no la más adecuada (desde el punto de vista profesional de la mujer) es el trabajo a tiempo parcial, para evitar la doble jornada laboral, y por tanto esta situación hace que el trabajo profesional de la mujer sea precario. Además se sigue considerando el trabajo que ella realiza como un complemento económico a los ingresos de la unidad familiar y no la principal fuente de ingresos de la misma. El Gráfico 2 muestra el índice de feminización de los contratos de trabajo registrados en Andalucía y en él se observa el elevado grado de feminización en los contratos a tiempo parcial.

Gráfico 2: Índice de feminización de los contratos de trabajo registrados en Andalucía según modalidad de los mismos

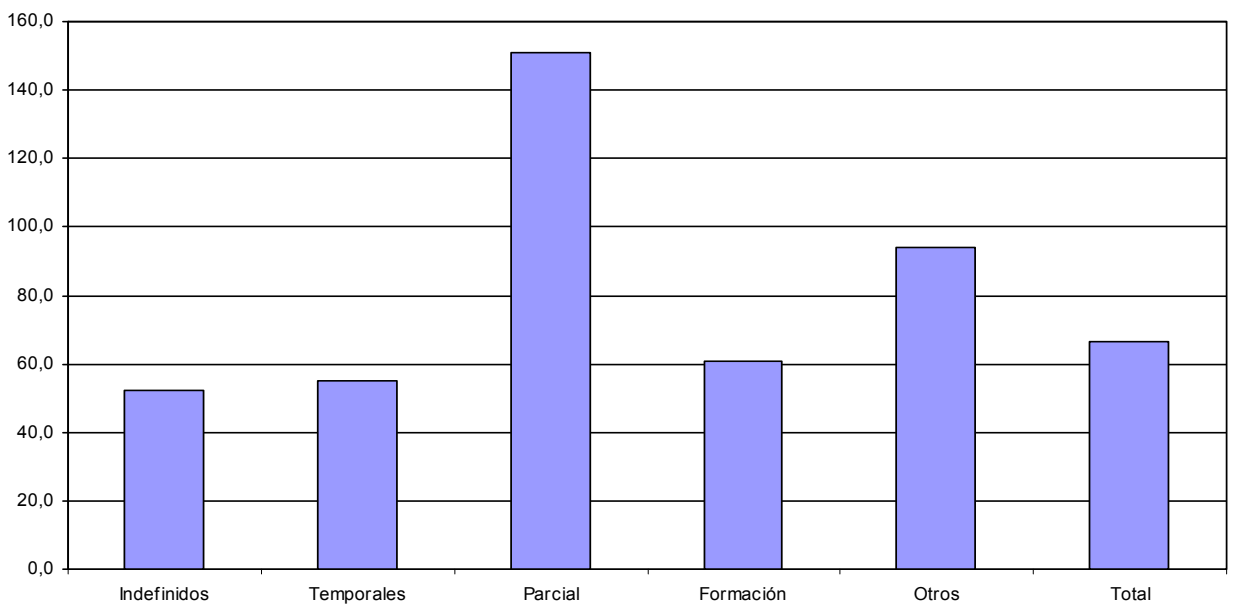

Fuente: IEA (Instituto de Estadística de Andalucía), año 2007.

\section{PERIODO 1995-2007}

3. EVOLUCIÓN DEL MERCADO DE TRABAJO EN ANDALUCÍA.

El incremento de la ocupación femenina de carácter estable en Andalucía en los últimos diez años, de 400.000 mujeres ocupadas en 1995 a más del doble (1.257.932) en el segundo trimestre de 2008

$7 \quad$ Número de mujeres por cada 100 hombres. 
(Gráfico 3) tiene un clara consecuencia en el impacto de las medidas de abaratamiento del coste del empleo femenino (contenidas en los dos últimos grandes Acuerdos de Concertación Social que se han firmado en esta región: el V Acuerdo de Concertación Social (Cavas 2003), vigente durante los años 2001, 2002 y 2003, y el VI Acuerdo (Castiñeira 2005), cuya vigencia comenzó en 2005 y se extenderá hasta 2008. Estos dos Acuerdos han supuesto un aumento de la contratación femenina a lo largo de dichos años donde a partir de mediados de los noventa el crecimiento del empleo es casi exponencial siendo la curva más acusada a partir de 2000, sobrepasando en 2004 el millón de mujeres andaluzas ocupadas.

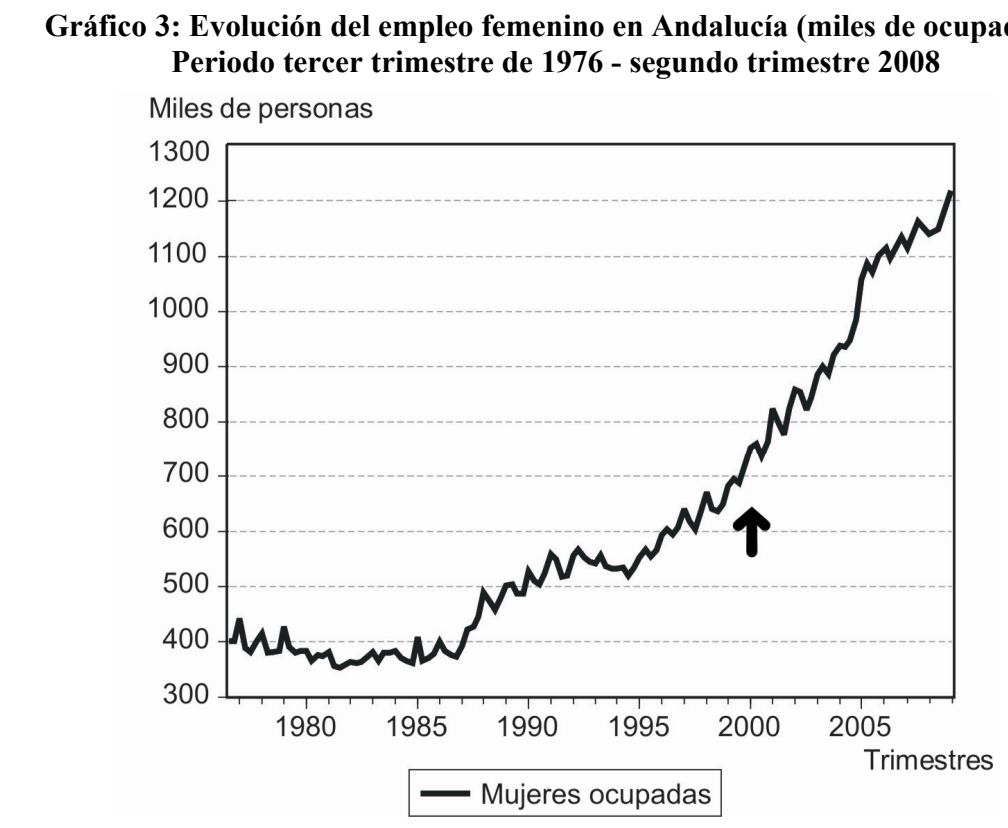

Fuente: Elaboración propia a partir de los datos del INE.

En el otro lado de la balanza, nos encontramos con el desempleo, el cual, en los años setenta indicaba que el número de mujeres inscritas como paradas en Andalucía era inferior al de los varones, pero a partir del segundo trimestre de 1999, esta cifra se invierte, manteniéndose esta tendencia en la actualidad. En el primer trimestre de 2008 el paro en el colectivo femenino es superior al masculino en casi más de 34.000 personas.

En el Gráfico 4 se puede observar como en ambos colectivos hubo una disminución del paro en 2001 y 2002. Posiblemente en ello 
influyesen medidas como el Decreto de 29 de julio de 1997 de la Consejería de Trabajo e Industria que aprobó programas de fomento del empleo ${ }^{8}$ o el Decreto 11/1999, de 26 de enero, de la Consejería de Trabajo e Industria donde se establecía incentivos a la contratación estable de mujeres mediante la reducción de la jornada laboral a 35 horas semanales, sustitución de vacantes y ausencias legales motivadas por maternidad, acogimiento y adopción, etc. Así, las medidas contempladas en esta norma posibilitaron crear más puestos de trabajo, a la vez que mejoraron las condiciones de trabajo de los trabajadores con responsabilidades familiares ${ }^{9}$ (Rodríguez y Pérez del Río 2005).

Gráfico 4: Evolución del paro en el colectivo femenino y masculino en Andalucía (miles de personas). Periodo 1976-2008

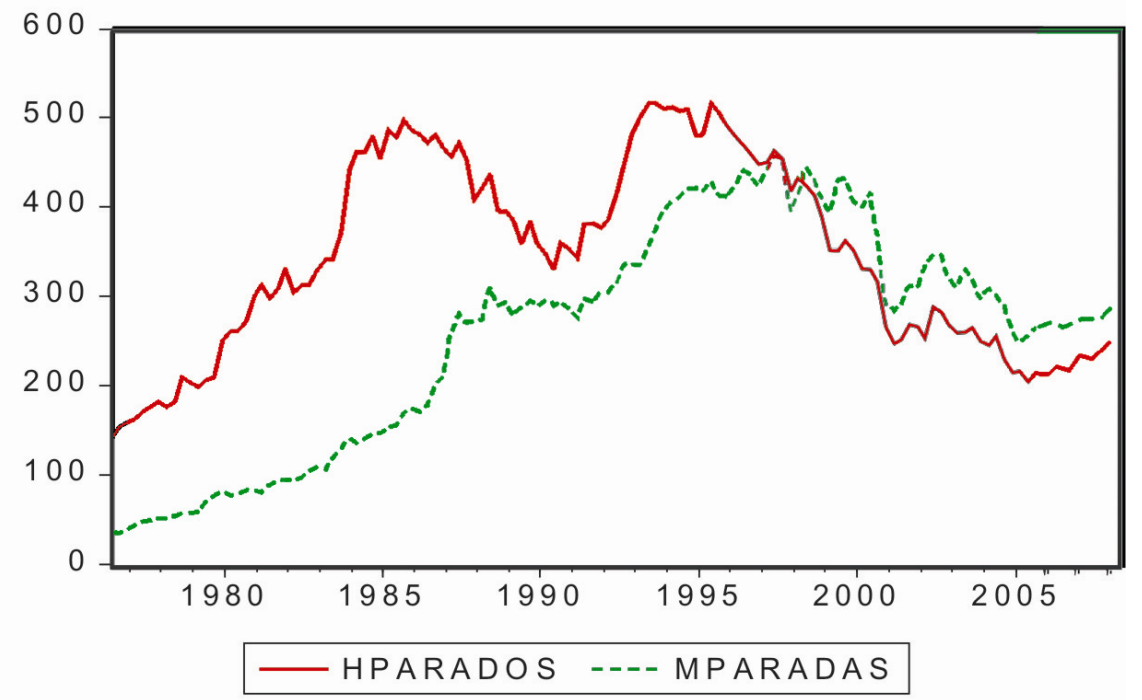

Fuente: Elaboración propia a partir de INE.

8 Este Decreto contiene medidas de apoyo al empleo de la mujer. En concreto, establece incentivos económicos al empleo estable femenino con una subvención a tanto alzado al empleador que podría llegar a 3.000 euros en determinadas circunstancias (contratos de nueva incorporación, cuando la empresa contrate a mujeres desempleadas que han realizado un curso de formación profesional ocupacional en los tres años anteriores a la contratación estrechamente relacionado en su contenido con la actividad a desarrollar en el puesto de trabajo, etc.).

9 Esta norma fue modificada por el Decreto 213/2003, de 15 de julio, durante la fase de evaluación del V Acuerdo de Concertación Social y derogada por el Decreto 149/2005, de 14 de junio, Decreto en el cual se priorizan las acciones implantadas en favor de determinados segmentos de la población, entre ellas, y en segundo lugar después de los jóvenes menores de treinta años, las mujeres desempleadas. 
A partir del año 2002 en el que se firma el Pacto Andaluz por la Economía social, donde hay un reconocimiento explicito de la representatividad de la CEPES-Andalucía como Agente Social se han conseguido logros importantes influyendo en la disminución del empleo, lo que ha motivado la continuidad de este acuerdo de concertación social con la firma del II pacto andaluz por la Economía Social, pero los avances más importantes en el campo del empleo se han obtenido a través de los acuerdos de Concertación Social que se detallan a continuación.

\subsection{Acuerdo de Concertación Social de Andalucía}

Entre los objetivos del V Acuerdo (Sánchez-Rodas 2005) se encontraba crear más empleo estable entre los colectivos más desfavorecidos, principalmente el femenino, apoyando para ello a las pequeñas y medianas empresas y promoviendo el espíritu y las iniciativas emprendedoras. Cabe destacar de este Acuerdo, como elementos novedosos, la implantación de criterios de modulación de la cuantía de los incentivos en materia de fomento estable del empleo femenino en función de la pertenencia a los colectivos más desfavorecidos y en función del tamaño de la empresa, beneficiando a las empresas de menores dimensiones ${ }^{10}$.

Como hemos indicado anteriormente, a partir de 2004 decae la vigencia del V Acuerdo, iniciándose una situación transitoria (que durará hasta mediados de 2005), en la que se suspenden algunas de las acciones encaminadas al fomento del empleo y se abre un proceso de evaluación del mencionado Acuerdo. La suspensión de estas medidas produjo un aumento del desempleo en el colectivo femenino y también en el masculino, como queda reflejado en el Gráfico 4 perjudicando el dinamismo y la evolución positiva del mercado laboral andaluz en su conjunto y, en particular, del femenino (se observa claramente un incremento del número de mujeres desempleadas en el primer trimestre de 2005).

\subsection{Acuerdo de Concertación Social de Andalucía}

Del análisis de la eficacia del $V$ Acuerdo, cabe resaltar que las medidas de fomento del empleo introducidas por él, si bien consiguieron

10 Asimismo, y en ejecución del V Acuerdo, la Consejería de Empleo y Desarrollo Tecnológico reguló, mediante Decreto de 1 de abril de 2003, los programas para la inserción laboral. 
una mejora tanto cuantitativa como cualitativa de la situación del mercado laboral andaluz, según los datos expuestos anteriormente, no se produjo una convergencia con el resto de las comunidades autónomas y con la Unión Europea.

El VI Acuerdo se firmó entre los actores sociales y el Gobierno andaluz en enero de 2005 y con vigencia hasta 2008. Entre los objetivos prioritarios se encuentra impulsar el empleo femenino, mediante la incorporación de la mujer al mercado laboral, elevando su tasa de actividad hasta niveles cercanos al $84 \%$ de la media de la Unión Europea y aumentando su ocupación en al menos un $20 \%$, mejorando la estabilidad en el empleo e incrementando en un $20 \%$ la contratación indefinida. Asimismo, se pretende ampliar el tejido empresarial andaluz hasta un mínimo de 500.000 empresas.

Por tanto, es objetivo prioritario del VI Acuerdo fomentar el empleo de la mujer y, por ello, en su aplicación se desarrollarán acciones de carácter específico, como se venía haciendo hasta el momento, incorporando aquellas líneas de trabajo en las que la evaluación de la experiencia comparada demuestre que están mejorando con el referente del entorno europeo, la posición y la participación de las mujeres en el mercado de trabajo. Estas medidas consisten básicamente en:

a) Incentivar la contratación estable de mujeres, atendiendo con carácter prioritario a los sectores en los que se encuentran subrepresentadas, las actividades y sectores de mayor índice de temporalidad y las situaciones singulares de la vida laboral de la mujer (como es la reincorporación al mercado laboral tras la maternidad), así como apoyar el mantenimiento y la creación de redes de empresarias.

b) Fomentar el autoempleo de mujeres, a través de medidas de creación de empleo que incidan sobre la oferta. Entre ellas, hay que destacar iniciativas de diversos tipos como el fomento de PYMES, el autoempleo, la economía social o los nuevos yacimientos de empleo.

3.3. Medidas asistenciales para favorecer la conciliación de la vida laboral y familiar

Las principales medidas se encuentran reflejadas en el Plan de Apoyo a las familias con rentas bajas de la comunidad autónoma de 
Andalucía que han optado por una fórmula de apoyo integral a este colectivo. Algunos de sus contenidos tocan de lleno aspectos relacionados con la conciliación de la vida laboral y familiar (Chinchilla y León 2004), si bien excede este ámbito y penetra en otros, muy relacionados, como el de la educación o la economía social. El mencionado Plan de Apoyo se ha plasmado en sucesivas iniciativas legislativas que arrancaron en 2002 a través del Decreto 137/2002, de 4 de mayo. Esta norma contiene un conjunto de disposiciones básicas dirigidas a satisfacer las necesidades de las familias andaluzas desde una perspectiva integral. Entre las medidas contempladas, relacionadas con el empleo de las mujeres y la conciliación de la vida laboral y familiar, cabe destacar las ayudas económicas por hijos, las ayudas para fomentar la reincorporación laboral de quiénes hayan interrumpido su actividad laboral para atender a sus hijos menores de tres años, siempre que no hayan transcurrido más de 48 meses desde el último nacimiento, o las ayudas para el cuidado de personas dependientes destinadas a la contratación de cuidadores.

Todas estas medidas suponen un cambio en las imágenes y en los modelos de realización de los hombres y las mujeres, y de los roles de ambos en los diferentes ámbitos de la vida personal, profesional y social (Beck-Gernstein et al. 2001). Por ello también será importante un cambio en la mentalidad del hombre y de la mujer andaluza, pues sino estas normas legales no tendrán la eficacia prevista.

\section{MATERIAL Y MÉTODOS}

Una vez analizadas las principales políticas activas de empleo femenino en Andalucía, realizaremos a continuación un análisis del mercado laboral femenino andaluz, con base en una investigación basada en un estudio de campo. En dicha investigación se va a utilizar modelos econométricos para estimar el "perfil" de la mujer andaluza trabajadora.

El estudio ha consistido en desarrollar un modelo Logit para estudiar la probabilidad de encontrar empleo por la mujer andaluza (ST Situación Laboral), atendiendo a sus características personales, basándonos en una encuesta realizada entre los meses de enero de 2006 y diciembre 2007 a 1.250 mujeres de las distintas provincias de la comunidad, y con una edad comprendida entre 16 y 65 años, seleccionando la muestra para que fuese representativa de la población. Las variables más relevantes tomadas en consideración son las siguientes: 
- Situación Laboral (ST) variable dicotómica tabulada con valores 1 que representa estar colocada y 0 estar desempleada.

- Estado civil: Subdividida en 4 variables dicotómicas siendo la de referencia la variable soltera (ecs), casada (ecc), separada (ecse), otros estados civiles, siendo ésta última variable la de referencia.

- Lugar de residencia (zona): Variable dicotómica tabulada con valores 1 que representa residir en una zona urbana y 0 residir en una zona rural.

- Edad.

- Sector en el que está ubicada su empresa: Sector primario ( $s p)$, sector secundario $(s s)$, sector terciario $(s t)$, siendo la variable de referencia el sector primario.

- Número de hijos $(n h)$.

- Afiliación seguridad social $(a f)$ : Variable dicotómica asignándole el valor 1 si está dada de alta en la seguridad social y 0 si trabaja sin seguridad social.

- Tipo de contrato $(t c)$ : variable dicotómica asignándole el valor 1 si es contrato fijo y 0 si es contrato temporal.

- Jornada laboral, subdividida en tres variables dicotómicas: completa $(j l c)$, media jornada $(j l m)$, otra modalidad (otra), siendo esta última variable, otra modalidad, la de referencia.

- Nivel Académico: Subdividida en 4 variables dicotómicas, sin estudios (nase), estudios básicos y medios (primaria, secundaria y bachiller) (naebm), estudios universitarios de grado medio (naum), estudios universitarios de grado superior (naus), siendo la variable nase la variable de referencia.

- Puesto que ocupa en la empresa subdividida en cuatro variables dicotómicas: obrera o similar $(o)$, administrativa o similar $(a)$; mando intermedio $(\mathrm{mi})$; cargo directivo $(\mathrm{cd})$; siendo la variable de referencia esta última.

- Salario neto al mes (sam).

- Número de empresas de economía Social de Andalucía (nes).

- Grado de satisfacción personal con el trabajo en casa y las obligaciones familiares $(s p)$.

- Tipo de empresa (te): variable dicotómica asignándole el valor $1 \mathrm{si}$ es empresa de economía social y 0 para el resto de las empresas. 
La representación matemática del modelo logit estimado es:

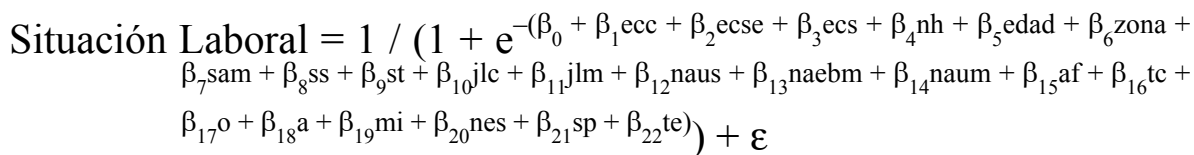

\section{RESULTADOS DE LA INVESTIGACIÓN}

En este epígrafe se presenta una descripción de la muestra de la población analizada, con base en la encuesta realizada a las 1.250 mujeres andaluzas, así como el Modelo Logit estimado para estudiar la probabilidad de encontrar empleo la mujer andaluza teniendo en cuenta determinadas características personales, siendo una de las variables explicativas del modelo, el tipo de empresa, si es de Economía Social o no.

Las características principales de la muestra expresadas en porcentaje son las siguientes:

- Lugar de residencia (zona): 63\% capital de provincia, 37\% resto de la provincia.

- Estado civil: $35 \%$ soltera (ecs), $52 \%$ casada (ecc), $11 \%$ separada (ecse), $2 \%$ otros.

- Edad: $13 \%$ entre 16 y 19 años, $18 \%$ entre 20 y 24 años, $26 \%$ entre 25 y 35 años, $26 \%$ entre 36 y 45 años, y $17 \%$ más de 45 . Se han establecido categorías diferentes a las de la EPA y similares a las leyes de fomento de empleo para comprender mejor la incidencia de éstas.

- Nivel Académico: 12,3\% sin estudios (nase), 69,6\% estudios básicos y medios (primaria, secundaria y bachiller) (naebm), 11,9\% estudios universitarios de grado medio (naum), 3,3\% estudios universitarios de grado superior (naus) y 3,8\% no contestan.

- Número de hijos $(n h): 2,4$ de media para las mujeres mayores de 35 años y 1,3 para las comprendidas entre 25 y 34 años.

- Situación laboral: desempleadas $68,2 \%$, trabajando 31,8\%.

- Afiliación laboral de las trabajadoras: dadas de alta en la Seguridad Social 77,6\%, sin Seguridad Social 22,4\%. 
- Tipo de trabajo: por cuenta propia 45,23\%, por cuenta ajena $54,8 \%$.

- Tipo de contrato: temporal $72,1 \%$, fijo $27,9 \%$.

- Jornada laboral: completa $(j l c)$ 49,2\%, media jornada $(j l m) 45,6 \%$, $5,2 \%$ otra modalidad.

- Puesto que ocupa en la empresa: obrera o similar $35,1 \%$, administrativa o similar $58,3 \%$, mando intermedio $2,1 \%$, cargo directivo $1,8 \%$, no contestan $2,7 \%$.

- Sector en el que está ubicada su empresa: Sector primario ( $s p$ ) 10,8\%, sector secundario (ss) 42,4\%, sector terciario (st) $46,8 \%$.

- Salario neto al mes (sam): inferior a 500 euros $29,4 \%$, entre 501 y 1.000 euros $50,6 \%$, entre 1.001 a 1.500 euros $12,3 \%$, entre 1.501 y 2.000 euros $5,0 \%$, más de 2.000 euros $2,7 \%$.

De las variables antes descritas las que tienen más relevancia sobre la probabilidad de estar colocadas son las que aparecen a continuación en el modelo:

$$
\text { Situación laboral }=\frac{1}{1+e^{-(u)}}+\varepsilon=\operatorname{Pr}[\text { empleada }]+\varepsilon
$$

siendo la función $u$ del modelo logit, la siguiente:

$$
\begin{aligned}
u=\beta_{0}+ & \beta_{1} \text { ecc }+ \\
\beta_{2} \text { ecse }+ & \beta_{3} \text { ecs }+\beta_{4} \text { nh }+\beta_{5} \text { edad }+\beta_{6} \text { zona }+\beta_{7} \text { sam }+ \\
& \beta_{8} \text { ss }+\beta_{9} \text { st }+\beta_{10 j} \text { lc }+\beta_{11} \text { jlm }+\beta_{12} \text { naus }+\beta_{13} \text { naebm }+ \\
& \beta_{14} \text { naum }+\beta_{15} \text { af }+\beta_{16} \text { tc }+\beta_{17} \mathrm{o}+\beta_{18} \mathrm{a}+\beta_{19} \text { mi }+\beta_{20} \text { nes }+ \\
& \beta_{21} \text { sp }+\beta_{22} \text { te }
\end{aligned}
$$

En el cuadro siguiente se muestran las estimaciones de los coeficientes descritos anteriormente. El modelo de partida contaba con 54 variables, pero en el modelo teórico descrito anteriormente y en el Cuadro 5 sólo aparecen las variables significativas así como sus desviaciones estándar. El cociente de ambos valores nos permitirá deducir si dicha variable influye en la probabilidad de encontrar empleo $(S T)$. Como se puede observar todas son significativas para $\alpha=0,05$. 
Cuadro 5: Estimación de Coeficientes del modelo Logit

\begin{tabular}{|l|cc|}
\hline Variable & Coeficiente estimado (Desviación) \\
\hline \hline Ordenada & $\mathrm{B}_{0}=0,1452$ & $(0,021)$ \\
\hline Estado civil casada $E C C$ & $\mathrm{~B}_{1}=-0,3782$ & $(0,017)$ \\
\hline Estado civil separada $E C S E$ & $\mathrm{~B}_{2}=0,5367$ & $(0,002)$ \\
\hline Estado civil soltera $E C S$ & $\mathrm{~B}_{3}=0,9854$ & $(0,237)$ \\
\hline Número de hijos $N H$ & $\mathrm{~B}_{4}=-1,1125$ & $(0,058)$ \\
\hline$E D A D$ & $\mathrm{~B}_{5}=-0,9874$ & $(0,358)$ \\
\hline$Z O N A$ & $\mathrm{~B}_{6}=0,3645$ & $(0,035)$ \\
\hline Salario neto al mes $S A M$ & $\mathrm{~B}_{7}=-11,5831$ & $(2,186)$ \\
\hline Sector secundario $S S$ & $\mathrm{~B}_{8}=0,4562$ & $(0,035)$ \\
\hline Sector terciario $S T$ & $\mathrm{~B}_{9}=1,1657$ & $(0,247)$ \\
\hline Jornada Laboral Completa $J L C$ & $\mathrm{~B}_{10}=-1,2515$ & $(0,282)$ \\
\hline Jornada Laboral Media $J L M$ & $\mathrm{~B}_{11}=3,9201$ & $(1,321)$ \\
\hline Nivel académico universitarios grado superior $N A U S$ & $\mathrm{~B}_{12}=3,2364$ & $(1,083)$ \\
\hline Nivel académico básicos y medios $N A E B M$ & $\mathrm{~B}_{13}=7,3897$ & $(1,245)$ \\
\hline Nivel académico universitarios medios $N A U M$ & $\mathrm{~B}_{14}=2,5677$ & $(0,687)$ \\
\hline Afiliación Seguridad Social $A F$ & $\mathrm{~B}_{15}=2,3571$ & $(0,287)$ \\
\hline Tipo de Contrato $T C$ & $\mathrm{~B}_{16}=-1,9664$ & $(0,265)$ \\
\hline Puesto de trabajo obrera $O$ & $\mathrm{~B}_{17}=2,6891$ & $(0,345)$ \\
\hline Puesto de trabajo administrativa $A$ & $\mathrm{~B}_{18}=3,8212$ & $(0,446)$ \\
\hline Puesto de trabajo mando intermedio $M I$ & $\mathrm{~B}_{19}=1,2561$ & $(0,445)$ \\
\hline Número de empresas de Economía Social $N E S$ & $\mathrm{~B}_{20}=0,0312$ & $(0,001)$ \\
\hline Satisfacción personal con el trabajo en casa $S P$ & $\mathrm{~B}_{21}=-4,526$ & $(0,356)$ \\
\hline Tipo de empresa $T E$ & $\mathrm{~B}_{22}=-0,2451$ & $(0,254)$ \\
\hline
\end{tabular}

$* \mathrm{R}_{\mathrm{MF}}^{2}=0,501$

* Parámetros significativos para $\alpha=0,05$

* Loglikehood ratio=112,2, p 00,002

Fuente: Elaboración propia.

\section{los siguientes resultados ${ }^{11}$ :}

De la estimación del modelo logit anterior podemos obtener

a) La mayoría de las variables seleccionadas influyen positivamente en la probabilidad de emplearse, siendo las variables que más relevancia tienen a estos efectos el nivel académico $\left(B_{13}=\right.$ $7,3897)$, la posibilidad de que la jornada no sea completa $\left(B_{11}=\right.$ $3,9201)$ y el puesto de trabajo que ocupa $\left(B_{18}=3,8212\right)$. Por el

11 Los coeficientes con signo positivo influyen favorablemente incrementado la probabilidad de que la mujer andaluza se encuentre colocada, los coeficientes con signo negativo disminuyen la probabilidad de que la mujer andaluza se encuentre colocada. 
contrario las variables que tienen una influencia negativa en la probabilidad de encontrar empleo serían el salario neto al mes $\left(B_{7}=-11,5831\right)$ y Satisfacción personal con el trabajo en casa $S P$ $\left(B_{21}=-4,526\right)$. A medida que la mujer andaluza se encuentra más satisfecha con las labores del hogar la probabilidad de encontrar empleo decrece, principalmente porque no lo busca, y es que en Andalucía todavía, sobre todo en las zonas rurales, predomina la cultura de que cuando la mujer contrae matrimonio y empieza a tener hijos debe "dejar" su empleo para dedicarse a las labores del hogar, siendo este su objetivo prioritario abandonando su carrera profesional en el mundo laboral.

b) El número de hijos continúa siendo una variable que influye negativamente para encontrar empleo en el colectivo femenino, con un coeficiente de $B_{4}=-1,1125$. A medida que se tienen más hijos la probabilidad de encontrar empleo decrece más rápidamente, y es que todavía es difícil conciliar la vida laboral y familiar a pesar de las normas dictadas por el gobierno.

c) El sector donde más probabilidad tienen de emplearse es en el terciario o de servicios $\left(B_{9}=1,1657\right)$, ya que en este sector se encuentran representadas las ramas de sanidad, enseñanza, limpieza y hostelería, que son los sectores donde el índice de feminización es mayor.

d) Las mujeres que viven en zonas urbanas tienen mayor probabilidad de encontrar trabajo que las que habitan en zonas rurales $\left(B_{6}=0,3645\right)$, ya que la mayoría de las empresas se encuentran en la zona urbana.

e) La mujeres que quiere trabajar en empresas de Economía Social tienen menor probabilidad de encontrar empleo que si lo hiciesen en otro tipo de empresas con distintas formas jurídica; esto se puede observar por el coeficiente negativo de la variable $\left(B_{22}=-0,2451\right)$.

f) El número de empresas pertenecientes a la Economía Social tiene una influencia muy leve en la probabilidad de encontrar empleo de una mujer andaluza debido al valor de su coeficiente que es muy cercano a cero $\left(B_{20}=0,0312\right)$.

A través de los resultados obtenidos con el modelo logit, se ha procedido a calcular las probabilidades que tiene de estar colocada la 
mujer andaluza basándonos principalmente en el numero de hijos y el estado civil (Cuadro 6). Podemos observar como el estado civil de la mujer y el número de hijos pueden influir negativamente en la probabilidad de estar colocada. Del modelo estimado se puede deducir que el $71,17 \%$ de las andaluzas solteras tienen probabilidad de estar colocadas frente al 43,11\% de las casadas. Es decir, existe una diferencia de más de 27 puntos en la probabilidad de las mujeres de estar trabajando según su estado civil. Esta diferencia tan acusada sólo es superada por Extremadura, con un $28,4 \%{ }^{12}$. Por tanto, podemos afirmar que todavía a principios del siglo XXI un elevado porcentaje de mujeres andaluzas abandonan el mercado laboral al casarse con la finalidad de atender las labores del hogar.

Cuadro 6: Probabilidad de estar colocada: variables relacionadas estado civil y número de hijos

\begin{tabular}{|l|c|c|c|}
\hline \multirow{2}{*}{ Estado civil } & \multicolumn{3}{|c|}{$\mathbf{N}^{\mathbf{0}}$ hijos } \\
\cline { 2 - 4 } & Ninguno & 2 hijos & 3 hijos \\
\hline Casada & $42,21 \%$ & $7,62 \%$ & $3,1 \%$ \\
\hline Soltera & $69,21 \%$ & $22,42 \%$ & $10,1 \%$ \\
\hline
\end{tabular}

Fuente: Elaboración propia. A partir de la estimación logística

Junto a los modelos logísticos, también se han utilizado tablas de contingencia donde se ha tratado de medir la asociación entre el nivel de educación, el estar trabajando o no y el estado civil. El instrumento de asociación utilizado ha sido el test chi-cuadrado procedente de la tabla de contingencia de asociación de las tres variables, tomando el valor 56,4 , con probabilidad 0 , lo cual indica que existe una fuerte asociación entre las tres variables objeto de estudio.

\section{CONCLUSIONES}

De las investigaciones desarrolladas y cuyos resultados se han mostrado con anterioridad podemos extraer como principales conclusiones los siguientes:

12 Según los primeros resultados provisionales de una investigación que estamos realizando en la actualidad sobre este tema. 
- La tasa de actividad femenina en Andalucía tiene una evolución creciente favorecida por las medidas de discriminación positiva.

- Se aprecia un ligero incremento en el empleo femenino en la Comunidad Autónoma Andaluza para el año 2008 como consecuencia de las medidas de fomento de empleo (Acuerdos de Concertación Social) dictadas por el gobierno autonómico, aunque no han conseguido paliar las desigualdades entre hombre y mujer respecto a tasas de actividad y paro siendo más elevadas las primeras en los hombres (actividad) y mayores las segundas (paro) en las mujeres.

- Existe una fuerte segregación por sexo en determinadas profesiones. Así, las mujeres siguen ocupando categorías profesionales con escasa cualificación, baja remuneración y poco prestigio social, el "techo de cristal" en el mundo laboral sigue siendo un handicap importante para el desarrollo profesional de la mujer andaluza, pues aún siendo su nivel de estudios, principalmente el universitario en mayor medida superior al hombre, ocupa por el contrario puestos menos relevantes en las empresas.

- La mayoría de los empleos temporales creados a lo largo de los últimos años (1996-2008) son ocupados por el colectivo femenino, siendo su situación laboral más precaria que la del colectivo masculino, al igual que el tipo de contrato, predominando en las mujeres los contratos a tiempo parcial.

- La cantidad y calidad de la incorporación de la mujer andaluza al trabajo remunerado está mediatizada por la cualificación y la formación profesional. A medida que aumenta el nivel de estudios se incrementa la tasa de actividad y de ocupación, disminuyendo, por tanto, el paro femenino. No obstante, a veces, esta disminución es debida al subempleo, ya que la mujer andaluza habitualmente ocupa puestos de trabajo por debajo de su categoría profesional y no acordes con su nivel de estudios o preparación académica.

- La tasa de empleo femenina se desploma en cuanto la mujer empieza a tener hijos. Así, sólo el $47,2 \%$ de las andaluzas trabajadoras con hijos menores de 12 años compatibilizan el trabajo a tiempo completo con la educación de los hijos, mientras que el $59,7 \%$ de las mujeres que no tienen niños pequeños trabajan a tiempo completo. A medida que se tienen más hijos la tasa de empleo femenino disminuye, influyendo también el estado civil. 
- Las desigualdades existentes entre hombres y mujeres en relación con el empleo, el desempleo y la remuneración, y conectadas con la asunción mayoritaria por parte de las mujeres de las responsabilidades familiares, son factores que subyacen en el problema del desempleo femenino.

- La normativa de fomento del empleo tiene una incidencia positiva moderada en el empleo femenino.

- El empleo femenino en las empresas de Economía social sigue siendo poco relevante, a pesar de ser la comunidad española con mayor número de empresas de este tipo.

- Las andaluzas que trabajan en empresas de Economía Social, según la encuesta realizada, ocupan puestos de poca responsabilidad, siendo muy similar al resto de las empresas en general.

- Una opción positiva para fomentar el empleo femenino en Andalucía sería crear cooperativas de trabajo, donde las mismas mujeres la autogestionen, ya que en muchas cooperativas andaluzas el mayor número de trabajadores está formado por el colectivo femenino pero los puestos directivos están ocupados por hombres.

Consideramos también que por las características propias de las entidades de Economía Social, la creación de empresas que pertenezcan a este sector puede ser una herramienta idónea para lograr una plena equiparación de condiciones y oportunidades laborales para hombres y mujeres. Las cooperativas en particular se crean en base a los valores de igualdad, equidad y solidaridad y por ello ha de ser intrínseco en ellas evitar situaciones de discriminación laboral por razón de sexo. Las cooperativas se son instrumentos válidos para el desarrollo profesional de las mujeres.

El que la tasa de empleo femenino incremente en Andalucía es una cuestión no sólo de políticas del gobierno (conciliación de la vida laboral y familiar, discriminación positiva, etc.) sino de concienciación de la sociedad y de cambio de mentalidad, la mujer no sólo se ha de desarrollar con el trabajo en el hogar sino también con una carrera profesional. 


\section{BIBLIOGRAFÍA}

ACEVEDO, Doris (2002) "Género y Políticas Laborales en un contexto de flexibilidad del trabajo, reestructuración productiva y precarización del trabajo", Salud de los trabajadores, Vol. 10, edición especial $n^{\circ} 1$, pp. 2-10.

AEAT (2006-2007), series homogéneas (en soporte informático), Madrid. www.aeat.es [http://www.meh.es/es-ES/Estadistica\%20e\%20Informes/ Indicadores\%20e\%20Informes\%20Macroeconomicos/Paginas/Indicadores \%20e\%20Informes\%20macroeconomicos.aspx].

APELQVIST, Katarina (1996) "Estrategias para una autoridad compartida entre hombres y mujeres en las cooperativas", Revista de la Cooperación Internacional, 1, pp. 35-47.

BARBERÁ, Esther; Maite SARRIÓ y Amparo RAmos (Coord.)(2000) Mujeres directivas: Promoción Profesional en España y el Reino Unido. Valencia: Institut Universitari d'Estudis de la Dona.

BAREA, José (1991) "La economía social en España", Revista de Economía y Sociología del Trabajo, 12, pp. 8-16.

- y José Luis Monzón (1996) Informe sobre la situación de las cooperativas y las sociedades laborales en España. Valencia: Ed. CIRIEC-España.

BeCK-GERnSHEIM, Elisabeth; Judith BUTLER y Lídia PUIGVERT (2001) Mujeres y transformaciones sociales. Barcelona: El Roure Editorial.

CARIDAD, José María (1998) Econometría, Modelos econométricos. Barcelona: Reverte.

- y Genoveva MiLlán (2007) "Modelización de las políticas activas de empleo femenino", Trabajo y Seguridad Social, 287, pp. 235-264.

CASTIÑEIRA, Jaime (2005) "El impacto de la normativa laboral sobre el empleo", Jornadas Universitarias Andaluzas de Derecho del Trabajo, Sevilla, CARL.

CAVAS, Faustino (2003) "El nuevo derecho del empleo. A propósito de la Ley 56/2003, de 16 de diciembre", Aranzadi Social, nº 19.

CEPES-Andalucía (2007a) Anuario Estadístico de CEPES Andalucía. Sevilla: Ed. CEPES-Andalucía [www.cepes-andalucia.es].

- (2007b) Informe Anual de la Economía Social Andaluza CEPES Andalucía. Sevilla: Ed. CEPES-Andalucía [www.cepes-andalucia.es].

CHAVEZ, M. Elena (1996) "El papel de la ACl en el progreso de la mujer en las cooperativas", Revista de la Cooperación Internacional, 1, pp. 51-62. 
CHINCHILLA, Nuria y Consuelo LEÓN (2004) La ambición femenina. Cómo reconciliar trabajo y familia. Madrid: Aguilar.

CONSEJERÍA DE INNOVACIÓN, CIENCIA Y EMPRESA (2007) El tercer sector. Análisis y perspectivas. Sevilla: Ed. Consejería de Innovación, Ciencia y Empresa. Junta de Andalucía [www.juntadeandalucia.es/innovacioncie nciayempresa/cocoon].

COSTA-PAU, Rosa (coord.) (2002) Les dones en l'àmbit del cooperativisme urbà. Barcelona: Fundació Roca i Galès.

GARRIGUES, Amparo (2004) La Maternidad y su consideración Jurídico-Laboral y de Seguridad Social. Madrid: CES.

InSTITUTO DE ESTAdÍSTICA DE ANDALUCía (1976-2008) Encuesta de Población Activa, series homogéneas (en soporte informático). Sevilla: www.iea.es [http://www.juntadeandalucia.es:9002/epa/index.htm].

InSTITUTO NACIONAL DE ESTADístICA (1976-2008) Encuesta de Población Activa, series homogéneas (en soporte informático). Madrid: www.ine.es [http: / / www.ine.es/jaxiBD/tabla.do?per=03\&type=db\&divi=EPA\&idtab $=276]$.

MILLÁN, Genoveva (1995) Modelos econométricos de demanda de Educación Superior y Empleo de los universitarios andaluces. Sevilla: Consejería de Educación y Ciencia, Junta de Andalucía.

MINISTERIO dE TRABAJO Y AsUNTOS SOCIALES (2008) Dirección general de la Economía Social, del Trabajo Autónomo y del Fondo Social Europeo. Bases de datos de la Economía Social [http://empleo.mtas.es/empleo/ economia-soc/BaseDeDatos].

MoLINA, Cristóbal (dir.) (2005) Empleo y Mercado de trabajo: nuevas demandas, nuevas políticas, nuevos derechos. Sevilla: CARL.

Monzón, José Luis y Jacques Defourny (dirs.) (1992) Economía Social. Entre economía pública y economía capitalista. Valencia: Ed. CIRIEC-España.

MORALES, José Manuel (2003) La comunitarización del empleo: su recepción por parte del Reino de España. Sevilla: CARL.

RIBAS, M. Antonia (2004) La Economía Social de cristal: un análisis de la situación de la mujer en la Economía Social. Tesis Doctoral. Universitat de Valencia.

- y Antonia SAJARDO (2005) "La diferente participación laboral de las mujeres entre las cooperativas y las sociedades laborales", Revista CIRIEC-España, 52, pp. 267-278.

Rodríguez, F. y T. PÉREZ del Río (2005) "Política de fomento de empleo de calidad", Empleo y Mercado de trabajo: Nuevas demandas, nuevas 
políticas, nuevos derechos, XXIII Jornadas Andaluzas de Derecho del Trabajo, Monografías de Temas Laborales, Sevilla: CARL, pp. 45-63.

SALDAÑA, Eva (2005) Discriminación retributiva en función del género, Monografías de Temas Laborales. Sevilla: CARL.

SÁNCHEZ-RODAS, Cristina (2005) "La dimensión local (comunidad, municipio) de la estrategia europea de empleo. Una aproximación desde la perspectiva de la legislación andaluza", Empleo y Mercado de trabajo: nuevas demandas, nuevas políticas, nuevos derechos, Sevilla: CARL, pp. 286-294.

WIRTH, Linda (2002) Romper el techo de cristal. Las mujeres en puestos de dirección. Madrid: Ministerio de Trabajo y Asuntos Sociales. 